\title{
Towards Isotope Ratio Measurement with an Electrometer Array on the Cameca IMS xf
}

\author{
Peter Williams*, Jan Lorincik*, Rick Hervig**, Laurie Leshin** \\ *Department of Chemistry \& Biochemistry \\ **Department of Geological Sciences \\ Arizona State University, Tempe, AZ 85287 \\ M. Bonner Denton, Roger Sperline \\ Department of Chemistry \\ University of Arizona, Tucson, AZ \\ Georges Slodzian \\ CSNSM, Bat. 104 \\ Universite Paris Sud \\ 91405 ORSAY Campus \\ France
}

The Genesis satellite has delivered back to Earth (somewhat more violently than was planned) samples of high-purity silicon and other materials implanted with ions from the solar wind. The objective is to determine isotope ratios of the various species blown out from the sun to identify the initial isotopic composition of the solar system - the baseline for understanding the myriad changes in isotope ratios that have resulted from biogenic and non-biogenic processing in the last 4.5 billion years. The objective of our work is to develop technology to determine the isotope ratio of oxygen in the solar wind.

The oxygen samples will be derived from collectors in the electrostatic concentrator of Genesis into which oxygen ions have been electrostatically accelerated to implant with energies $\sim 60 \mathrm{keV}$ and doses ranging from $\sim 3 \times 10^{14} \mathrm{~cm}^{-2}$ for ${ }^{16} \mathrm{O}$ to $1.2 \times 10^{11} \mathrm{~cm}^{-2}$ for ${ }^{18} \mathrm{O}$. The approach with the highest sensitivity to analyze these samples is secondary ion mass spectrometry (SIMS), using $\mathrm{Cs}^{+}$primary ions to produce high ionization efficiency for $\mathrm{O}^{-}$ion production. However, not only are the dose levels low but also because the oxygen is implanted, the signals will vary quite strongly - by $\sim 60 \%$ over a $100 \mathrm{~A}$ depth at the steepest. For accurate measurement it is clear that the normal isotope ratio methodology, of switching the mass spectrometer cyclically between the different oxygen isotopes, cannot yield accurate measurements and instead we need to devise a method of integrating all three ion signals over the entire implant profile. To date, multiple ion collection in Cameca, Inc 1270/1280 magnetic secondary ion mass spectrometers has involved siting separate small electron multipliers along the mass spectrometer direction-focal plane. Electron multipliers have numerous problems when accurate isotope ratios are required, and in addition the focusing for off-axis ions at the direction-focal plane has significant aberrations because ions of different energies are not focused to the same point at off-axis positions. 
To address these issues we have incorporated a novel microfabricated electrometer array into a Cameca IMS $3 \mathrm{f}$ secondary ion mass spectrometer. We have further modified the ion optics at the exit of the mass spectrometer so that the off-axis ${ }^{16} \mathrm{O}$ and ${ }^{18} \mathrm{O}$ ions are deflected back towards the optic axis; this allows us to minimize the size, and thus importantly the stray capacitance, of the electrometer array. In addition, the deflection optics allow us to correct the off-axis ion beams for energy dispersion and to move the direction focus points so that energy and direction focusing is achieved for all three isotopes. Fig. 1 is a SIMION ion optical simulation showing how a combination of quadrupole and dipole (deflector) elements causes the off-axis beams to converge and be focused onto the exit slit plane where the central ${ }^{17} \mathrm{O}$ beam is also fully focused.

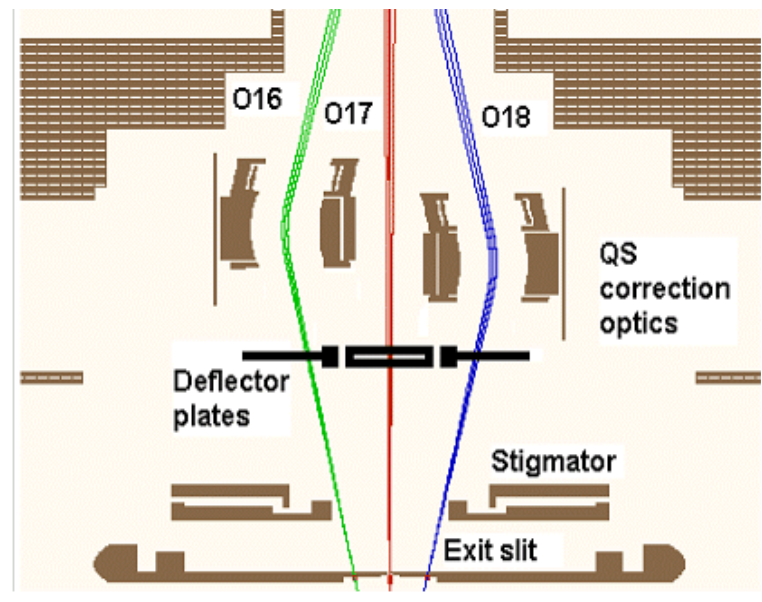

Figure 1. SIMION ion optical simulation of Cameca IMS $3 f$ showing modified exit optics (expanded horisontally to show detail)

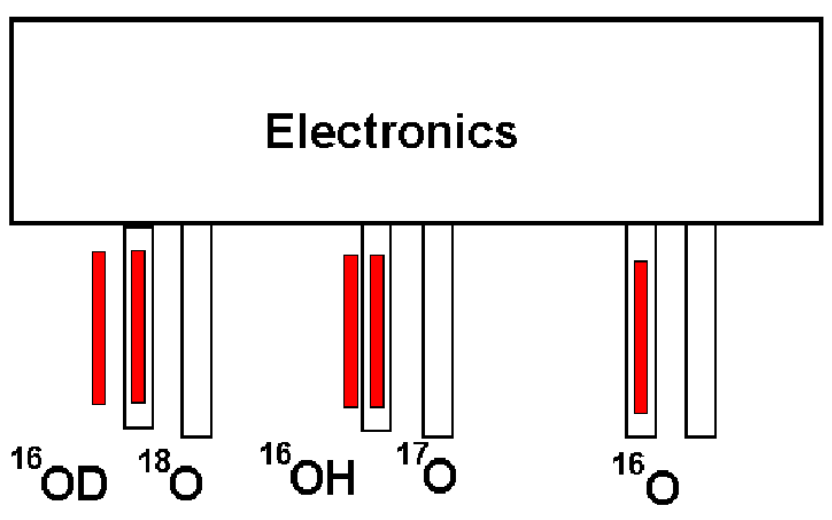

\section{Collector "fingers" (View from magnet)}

Figure 2. Layout of collector "finger" array showing how interfering ion beams are allowed to pass through the device.

Fig. 2 shows the construction of the electrometer array. The array consists of 3 pairs of collector "fingers", microfabricated out of silicon. One electrode of each pair is used to capture the incident oxygen ions while interfering species such as ${ }^{16} \mathrm{OH},{ }^{16} \mathrm{OD}$ are allowed to pass by; the second electrode serves as a reference and allows correction for noise effects. Furthermore, the pairs of fingers are spaced to serve as conventional slits so that the mass spectrometer can be tuned and focused using the conventional single electron multiplier detector. The performance objective for the electrometer is a noise level approaching 1-2 ions/second. In initial trials, a first-generation device had a detection limit of $\sim 25$ ions/second; the next-generation device that will be used for measurement is anticipated to be about an order of magnitude better.

Work supported by NASA SRLIDAP Program. 